\title{
Performance of a Slantlet Based OFDM Transceiver under Different Channel Conditions
}

\author{
Dr. Abbas Hasan Kattoush \\ Electrical Engineering department of Tafila Technical University - Jordan \\ Email:akattoush@yahoo.ca \\ Dr. Qasaymeh M. M. \\ Electrical Engineering department of Tafila Technical University - Jordan \\ Email: qasaymeh@ttu.edu.jo
}

\begin{abstract}
A major goal of the next-generation wireless communication systems is the development of a reliable high-speed wireless communication system that supports high user mobility. Orthogonal Frequency Division Multiplexing (OFDM) system is one of the most promising technologies for current and future wireless communications that has drawn a lot of attention. OFDM usually achieved by Fast Fourier Transform (FFT). In this paper, Fast Fourier Transform (FFT) is replaced by SlantLet Transform (SLT) in order to reduce Inter-Carrier Interference (ICI), Inter-symbol Interference (ISI), and to improve the bandwidth efficiency by removing the Guard Interval (GI) needed in FFT-OFDM. The new structure was tested and compared with conventional FFT-based OFDM for Additive White Gaussian Noise (AWGN) channel, Flat Fading Channel (FFC), and multi-path Selective Fading Channel (SFC). Simulation tests were generated for different channels parameters values. The obtained results showed the proposed system has an improved Bit Error Rate (BER) performance compared with the reference system. For SFC the SLT-OFDM performs better than the FFT-OFDM on the lower SNR region, while the situation reverses with increasing SNR values.
\end{abstract}

Index Terms-Slantlet Transform, Slantlet based OFDM, DWT based OFDM, Multi-path selective fading channel.

\section{Introduction}

Orthogonal Frequency Division Multiplexing (OFDM) system is one of the most promising technologies for current and future wireless communications. It is a form of multi-carrier modulation technologies where data bits are encoded to multiple sub-carriers, while being sent simultaneously ${ }^{[1]}$. Each sub-carrier in an OFDM system is modulated in amplitude and phase by the data bits. Modulation techniques typically used are binary phase shift keying, Quadrature Phase Shift Keying (QPSK), Quadrature Amplitude Modulation (QAM). The process of combining different sub-carriers to form a composite time-domain signal is achieved using FFT and inverse FFT operations ${ }^{[2]}$.

Manuscript received November 21 2011; revised January 10 2012; accepted January 302012.

corresponding author: Qasaymeh M. M. .
The main problem in the design of a communications system over a wireless link is to deal with multi-path fading, which causes a significant degradation in terms of both the reliability of the link and the data rate ${ }^{[3]}$. Multi-path fading channels have a severe effect on the performance of wireless communication systems even those systems that exhibits efficient bandwidth, like OFDM. There is always a need for developments in the realization of these systems as well as efficient channel estimation and equalization methods to enable these systems to reach their maximum performance ${ }^{[4]}$. The OFDM receiver structure allows relatively direct signal processing to combat channel delay spreads, which was a prime motivation to use OFDM modulation method in several standards ${ }^{[5-8]}$.

In transmissions over a radio channel, the orthogonality of the signals is maintained only if the channel is flat and time-invariant, channels with a Doppler spread and the corresponding time variations corrupt the orthogonality of the OFDM sub-carrier waveforms ${ }^{[9]}$. In a dispersive channel, self-interference occurs among successive symbols at the same subcarrier casing ISI, as well as among signals at different sub-carriers casing ICI. For a time-invariant but frequency-selective channel, ICI, as well as ISI, can effectively be avoided by inserting a cyclic prefix before each block of parallel data symbols at the cost of power loss and bandwidth expansion ${ }^{[2]}$.

Conventional OFDM/QAM systems are robust for multipath channels due to the cyclically prefixed guard interval which is inserted between consequent symbols to cancel ISI. However, this guard interval decreases the spectral efficiency of the OFDM system as the corresponding amount ${ }^{[10]}$. Thus, there have been several approaches of wavelet-based OFDM which does not require the use of the guard interval ${ }^{[11-18]}$. It is found that OFDM based on Haar orthonormal wavelets (DWTOFDM) are capable of reducing the ISI and ICI.

Recently, Selesnick ${ }^{[19]}$ has constructed a new orthogonal discrete wavelet transform, the Slantlet transform (SLT), with two zero moments and improved time localization ${ }^{[20]}$. SLT transform found important applications in signal and image processing; it has been successfully applied in image compression and de noising. It also retains the basic characteristic of the 
usual filter-bank such as octave band characteristic, a scale dilation factor of two and efficient implementation. However, the SLT is based on the principle of designing different filters for different scales unlike iterated filterbank approaches for the DWT ${ }^{[21]}$.

In this paper, SLT found a new application; it is used as a main building block in designing an improving the spectral efficiency of an OFDM system where the SLT modulator replaces the FFT modulator. Simulation results show that proposed system has better performance than conventional Fourier based OFDM under different channel conditions.

\section{Slantlet Transform}

The Slantlet transform is an orthogonal discrete wavelet transform with two zero moments and with improved time localization ${ }^{[19]}$. It uses a special case of a class of bases described by Alpert in ${ }^{[22]}$, the construction of which relies on Gram-Schmidt orthogonalization. SLT is based on the principle of designing different filters for different scales unlike iterated filter-bank approaches for the DWT. Selesnick described the basis from a filter-bank viewpoint, gave explicit solutions for the filter coefficients, and described an efficient algorithm for the transform.

The usual iterated DWT filter-bank and its equivalent1 form are shown in Fig. 1. The "slantlet" filter-bank is based on the equivalent structure that is occupied by different filters that are not products. With this extra degree of freedom obtained by giving up the product form, filters of shorter length are designed satisfying orthogonality and zero moment conditions.

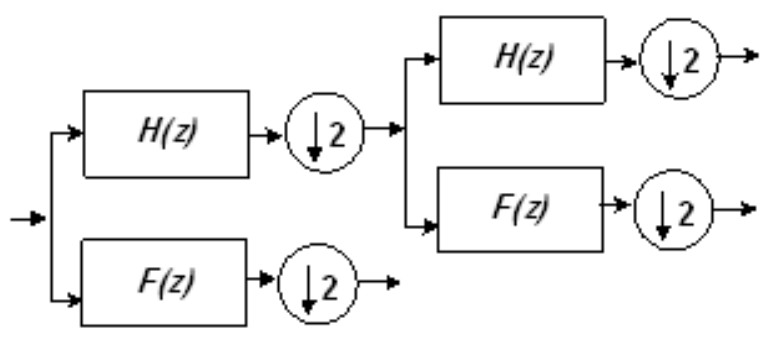

(a) Two-scale iterated filter-bank

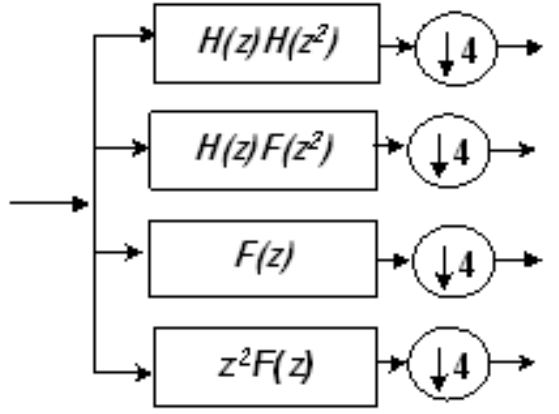

(b) Equivalent form using the DWT

Fig. 1: Two-scale iterated filter-bank and its equivalent form using the DWT.

For two-channel case the Daubechies filter ${ }^{[23]}$ is the shortest filter which makes the filter-bank orthogonal and has $K$ zero moments. For $K=2$, zero moments, filters $H(z)$ and $F(z)$ are of length 4 . For this system, the iterated filters in Fig. 1 are of length 10 and 4. Without the constraint that the filters are products, an orthogonal filter-bank with $K=2$ zero moments can be obtained where the filter lengths are 8 and 4 . That is a reduction by two samples, which is a difference that grows with the number of stages. This reduction in length, while maintaining desirable orthogonality and moment properties, is possible because these filters are not constrained by the product form arising in the case of iterated filter-banks.

The filters coefficients used in the SLT filter-bank as derived in ${ }^{[19]}$. Some characteristic features of the SLT filter-bank are orthogonal, having two zero moments and has octave-band characteristic. Each filter-bank has a scale dilation factor of two and provides a multiresolution decomposition. The Slantlet filters are piecewise linear. Even though there is no tree structure for SLT, it can be efficiently implemented like an iterated DWT filter-bank ${ }^{[19]}$. Therefore, a computational complexity of the SLT is of the same order as that of the DWT. 


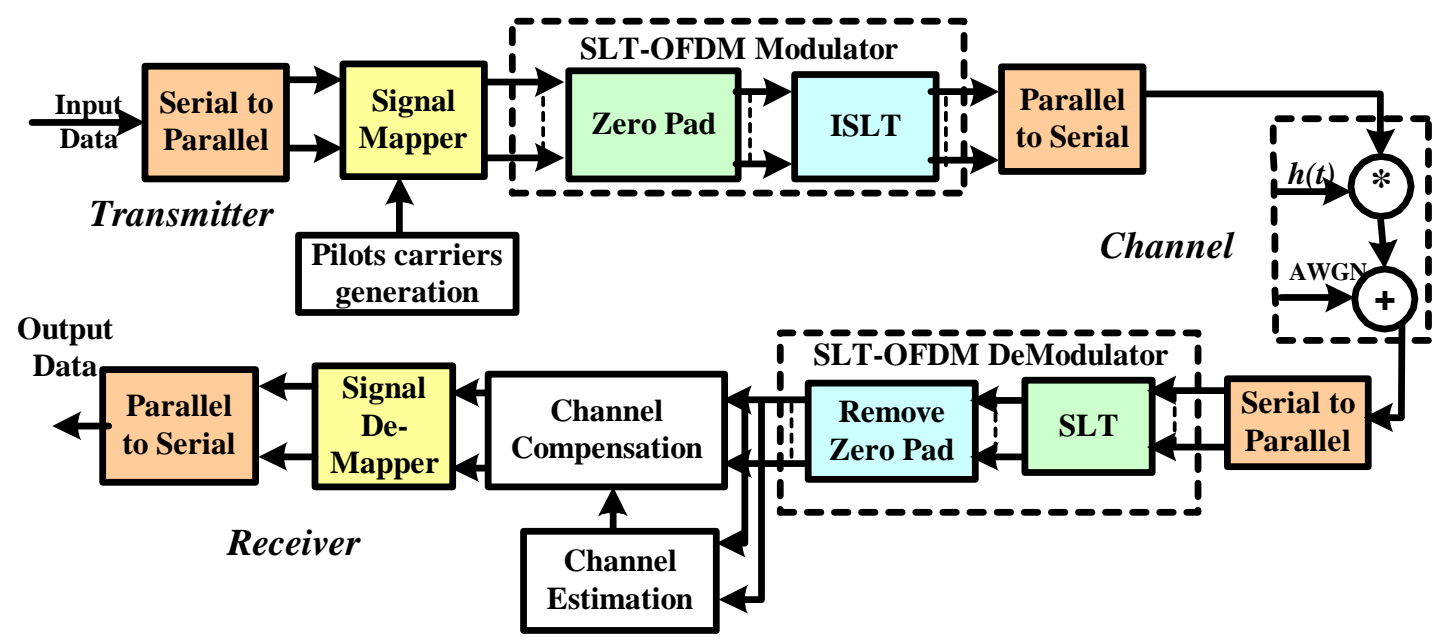

Fig. 2: Block Diagram of SLT-OFDM System

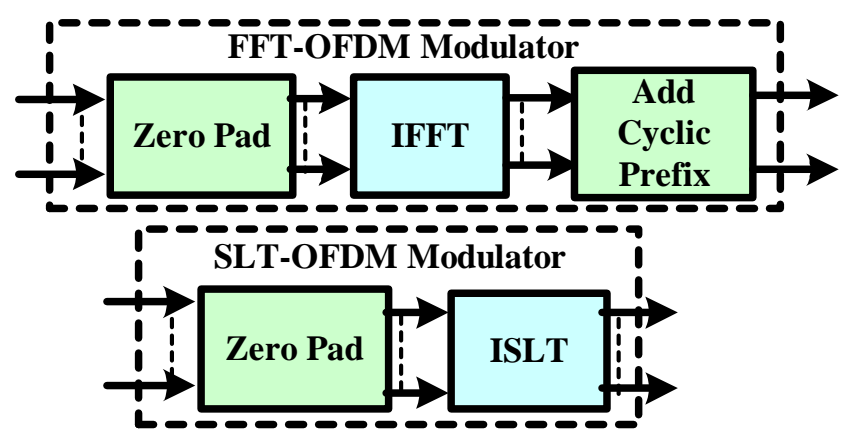

Fig. 3: FFT-OFDM and SLT-OFDM modulators

\section{Proposed System for Slantlet Transform -OFDM}

In the previous section, the SLT computation algorithm was provided that gains much improvement in terms of low computational complexity. The block diagram of the proposed system for OFDM is depicted in Fig. 2 It is very similar to that of FFT-OFDM; the only differences are in the OFDM modulator-demodulator blocks. The processes of serial to parallel (S/P) converter, signal demapper, and insertion of training sequence are the same as in the FFT-OFDM system. The SLT-based OFDM modulator consists of zero padding and inverse SLT (ISLT) blocks while the SLT-based OFDM demodulator consists of SLT and zero-pad removal blocks as shown in Fig. 2. The main difference between FFT-OFDM and SLT-OFDM is that in SLT-OFDM there is no need for adding a cyclic prefix to OFDM symbols as shown in Fig. 3; therefore the spectral efficiency and the data rate of SLT-OFDM are better than those of FFT-OFDM.

\section{Results and Discussion of Proposed System Simulations}

In this section the simulations of the proposed SLTOFDM system are provided using MATLAB. The bit error rate performance of the OFDM system is simulated for different channel models: additive white Gaussian noise (AWGN) channel, flat fading channel (FFC), and selective fading channel (SFC). In this simulation BPSK is used, the number of the sub-carriers is 64 and, the bandwidth used was $5 \mathrm{MHz}$. All simulation results are provided as a function of signal-to-noise-ratio (SNR) defined as the ratio of signal power to noise power and usually expressed in $\mathrm{dB}$. 


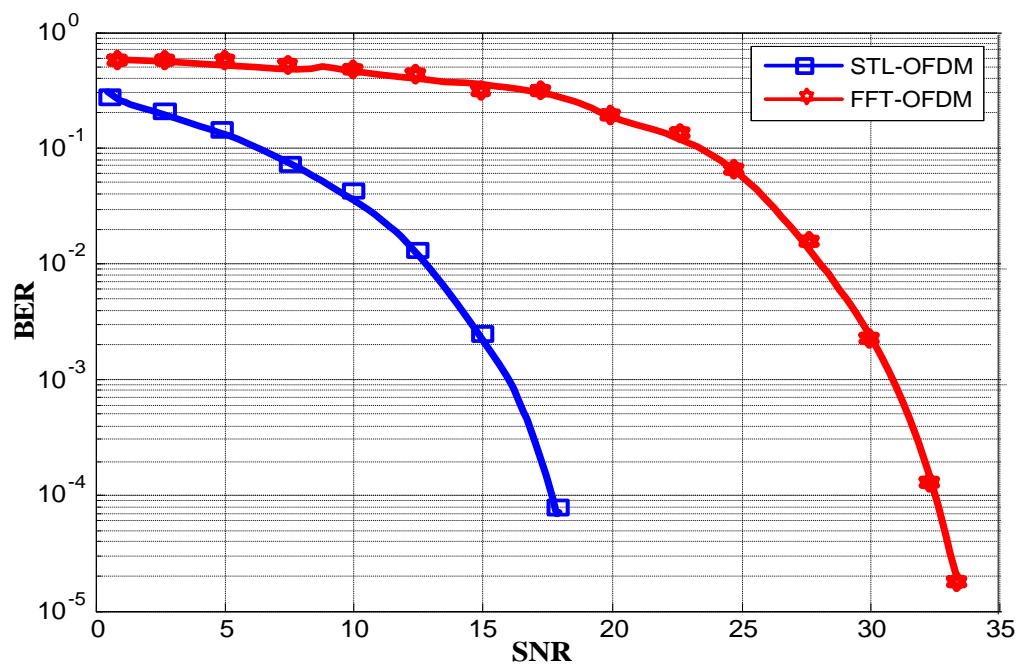

Fig. 4: BER performance of SLT-OFDM in AWGN channel model.

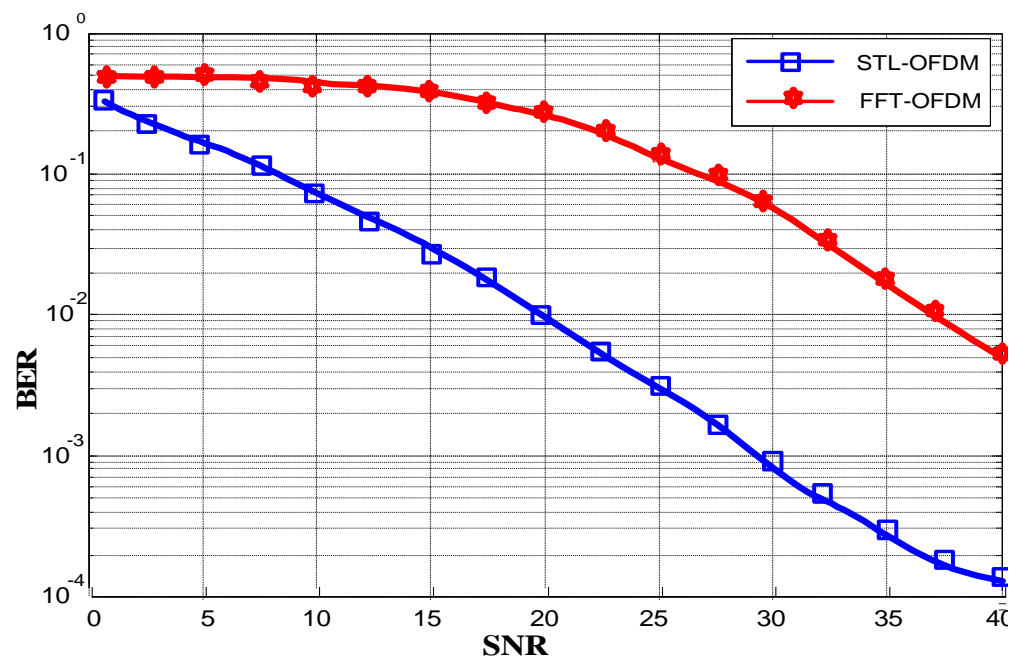

Fig. 5: The BER performance of SLT-OFDM in FFC at Max. Doppler Shift=5Hz.

\section{a. Performance of SLT-OFDM in AWGN Channel Environment}

Fig. 4 shows the results of simulation for BER performance of SLT-OFDM in AWGN channel. It is clearly seen that SLT-OFDM has much better performance than FFT-OFDM. For example to have $\mathrm{BER}=10-3 \mathrm{SLT}-\mathrm{OFDM}$ requires $16.5 \mathrm{~dB}$ SNR, while FFT-OFDM requires $31.5 \mathrm{~dB}$ SNR. This reflects the fact that the orthogonality of the SLT-OFDM sub-carrier waveforms is much better than that for FFT-OFDM. Also the efficiency of SLT-OFDM increases with decreasing the required value of BER for such type of channels.

\section{b. Performance of SLT-OFDM in a Flat Fading Channel}

In this channel, in addition to AWGN all frequency components of signal are affected by a constant attenuation and a linear phase distortion, which has been chosen to have a Rayleigh distribution. Fig. 5 shows BER performance of SLT-OFDM in FFC at maximum Doppler Shift equal $5 \mathrm{~Hz}$. From Fig. 5 it is seen that to have $B E R=10^{-2}$ the required SNR for SLT is about $20 \mathrm{~dB}$, while for FFT-OFDM it is required 


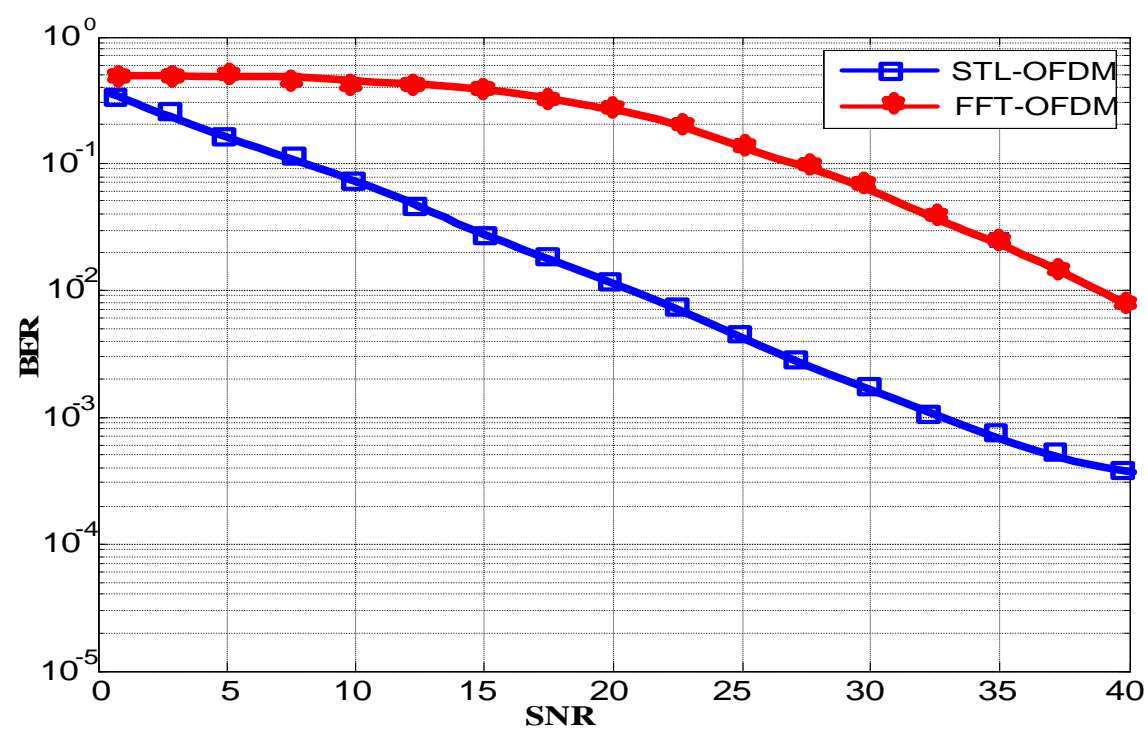

Fig. 6: The BER performance of SLT-OFDM in FFC at Max. Doppler Shift=500Hz.

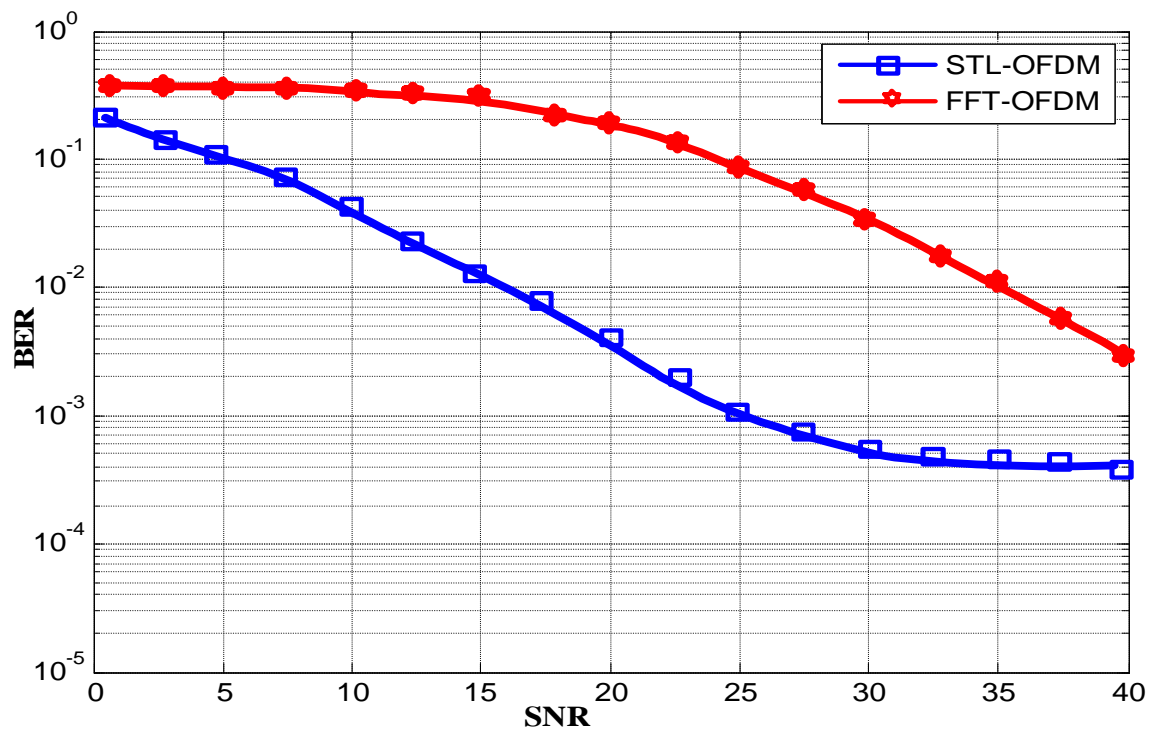

Fig. 7: The BER performance of SLT-OFDM in FFC at Max. Doppler Shift $=1100 \mathrm{~Hz}$

equal $500 \mathrm{~Hz}$, and $1100 \mathrm{HZ}$ are found. Fig.

$37 \mathrm{~dB}$ of SNR. So SLT-OFDM has about $17 \mathrm{~dB}$ gain improvement over FFT-OFDM. In general SLT- OFDM significantly increases SNR improvement and has a big advantage over the conventional system for this channel model. Other Doppler shifts are used for simulations in FFC, and the BER performance of SLT- OFDM and FFT-OFDM in FFC at maximum Doppler shifts values
6 provides BER performance of the two types of OFDM at maximum Doppler shift value equal $500 \mathrm{~Hz}$ and Fig. 7 depicts BER performance of the two types of OFDM at maximum Doppler shift value equal $1100 \mathrm{~Hz}$. For the three cases of Doppler shift, SLT-OFDM has better performance than the conventional. 


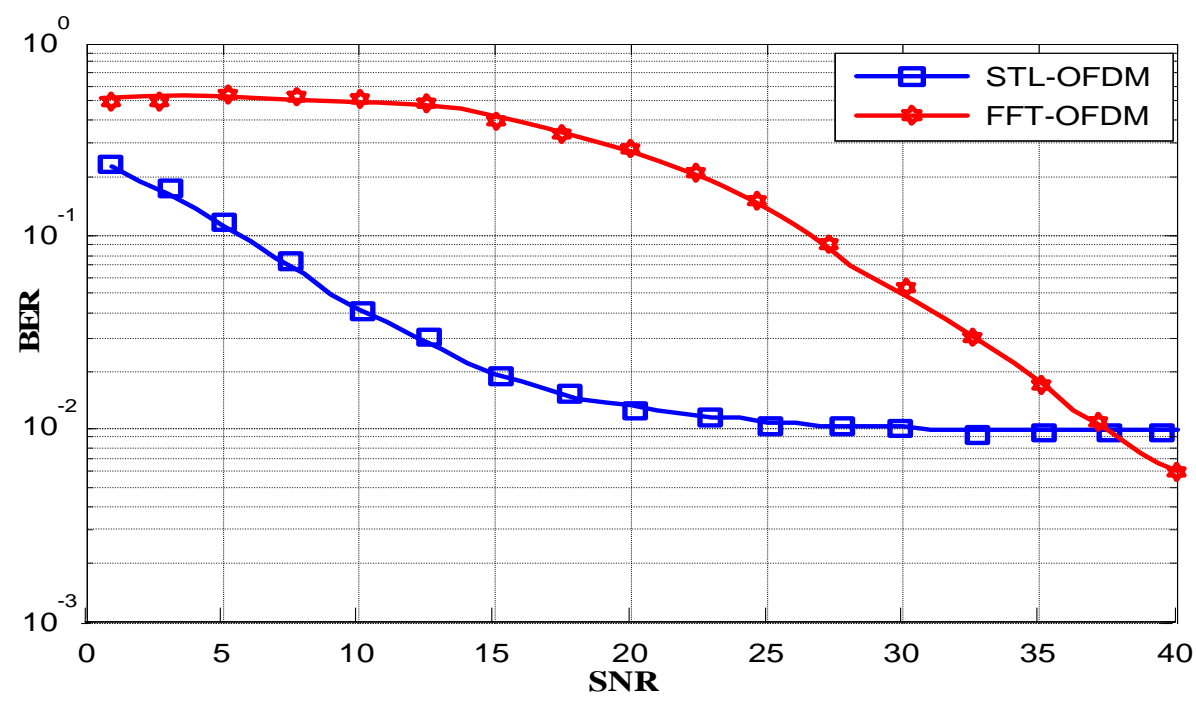

Fig. 8: The BER performance of SLT-OFDM in SFC at max. Doppler Shift=5Hz.

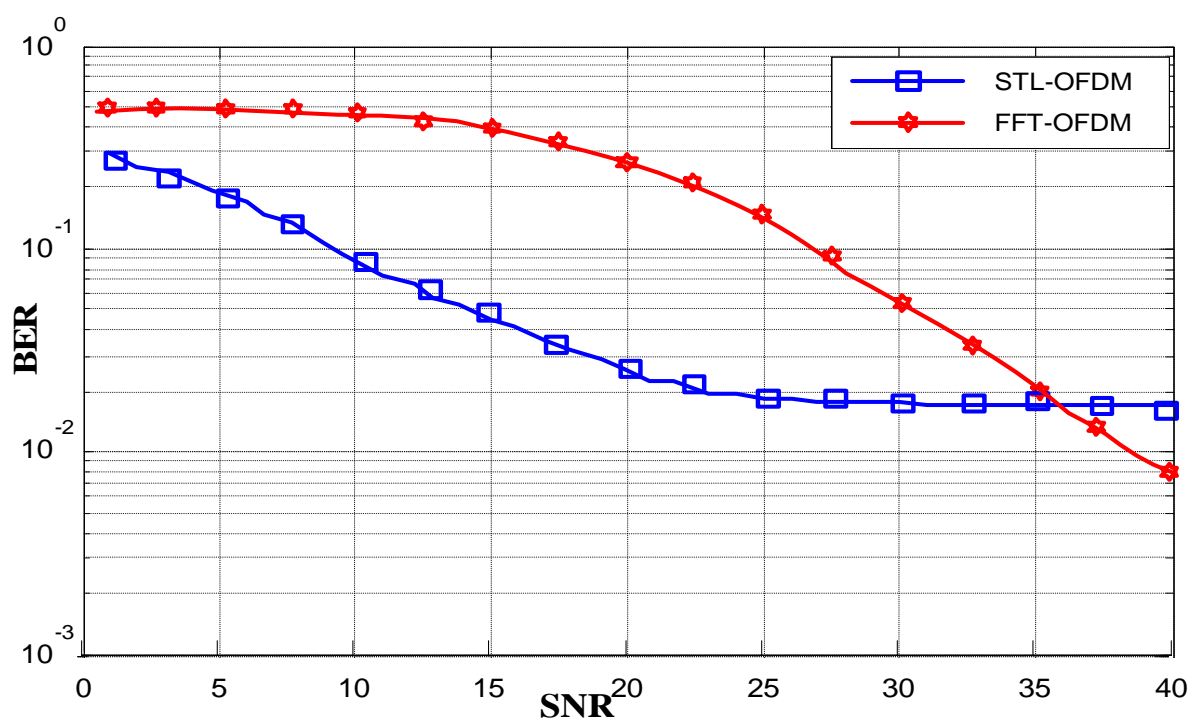

Fig. 9: The BER performance of SLT-OFDM in SFC at maximum Doppler Shift $=500 \mathrm{~Hz}$

\section{c. BER Performance of SLT-OFDM in Selective Fading Channel}

In this section, the channel model is selected to be selective fading channel, where the parameters of the second ray channel is assumed with a second path gain of $-10 \mathrm{~dB}$, and a second path delay of one sample. Fig. 8 clearly shows that the BER performance of SLT-OFDM in this case is also better than that for FFT-OFDM. SLTOFDM has BER performance equal $10-2$ at $\mathrm{SNR}=30 \mathrm{~dB}$ and the FFT-OFDM has the same BER performance at 38dB. BER performance of SLT-OFDM becomes constant after a certain SNR. From these results it can be concluded that SLT-OFDM has better performance than that of FFT-based OFDM in the different channels that have been studied. The two systems were simulated and tested in SFC for $500 \mathrm{~Hz}$ and $1100 \mathrm{~Hz}$ maximum Doppler shift. The results of BER performance for the two cases are given in Fig. 9 and Fig. 10. From simulation results it can be concluded that SLT-OFDM as DWT-OFDM is very sensitive to Doppler shift variations and its performance decreases with increasing Doppler shift 


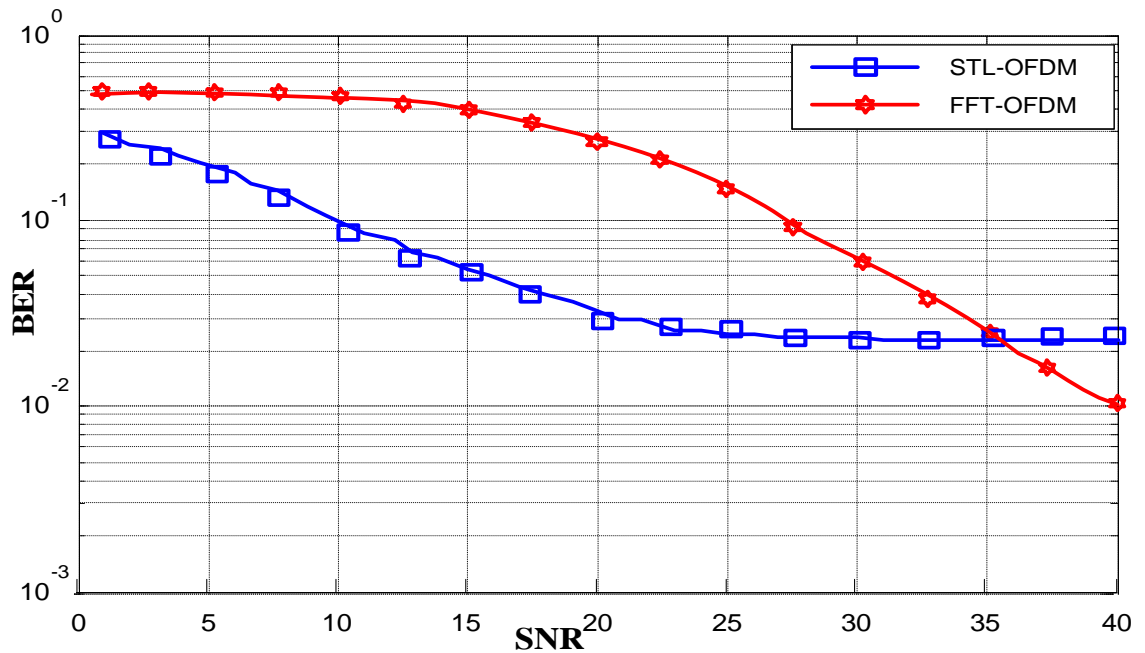

Fig. 10: The BER performance of SLT-OFDM in SFC at max. Doppler Shift=1100Hz.

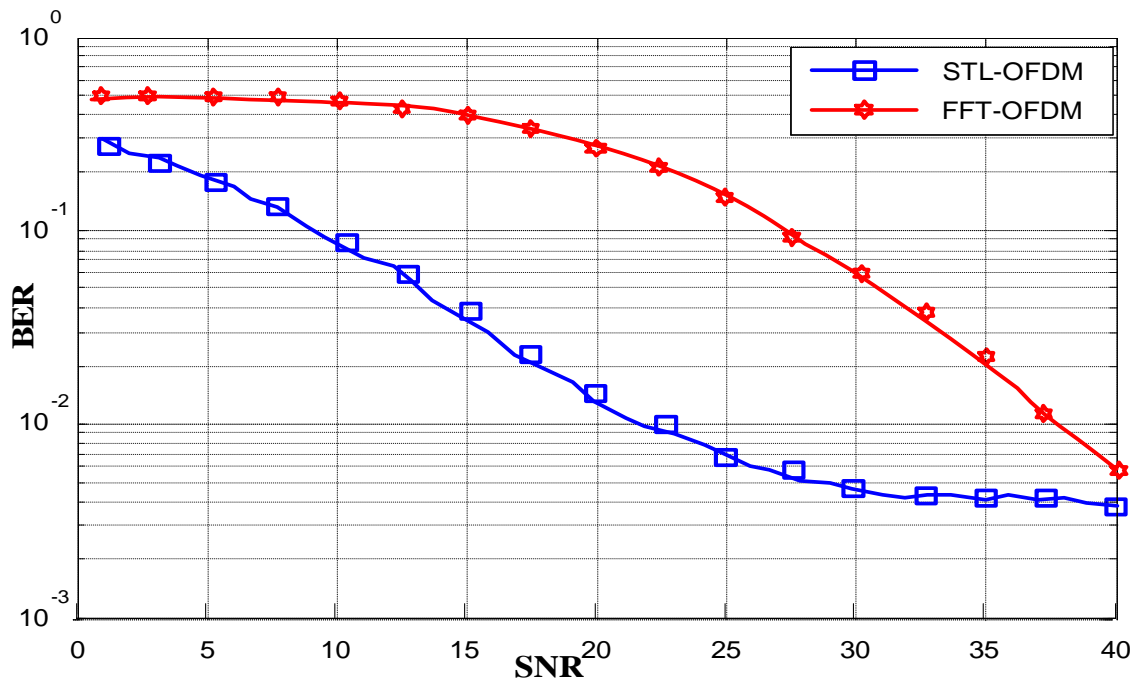

Fig. 11: The BER performance of SLT-OFDM in SFC at second Path Gain= $-20 \mathrm{~dB}$.

Now different values of second path gain are used to study the effect of second path gain on BER performance of the two systems. The simulation results for second path gains equal $-20 \mathrm{~dB},-11 \mathrm{~dB},-5 \mathrm{~dB}$, and $1 \mathrm{~dB}$ are provided Fig.11, Fig, 12, Fig,13, and Fig.14. Depending on results provided in Fig. 8 and Fig. 11 for gains $-10 \mathrm{~dB}$ and $-20 \mathrm{~dB}$ respectively, and for maximum Doppler Shift $=5 \mathrm{~Hz}$, SLT-OFDM still has better performance than FFT-OFDM. The behaviour of BER performance of SLT-OFDM in selective fading channel is similar to that of DWT-OFDM; it is very sensitive to Doppler frequency variations. Depending on the channel second path gain and delay there is a critical value of Doppler frequency for which increasing the SNR does not affect the BER performance of the system. This is produced by the loss in orthogonality between the carriers as a result of the multipath wireless channel. SLT-OFDM system has better performance than conventional system; however for Doppler frequencies exceeding critical value FFT-OFDM outperforms proposed system for very high SNR values (SNR > 40dB) since increasing signal power does not solve the problem of interference. The Doppler frequency critical value increases with decreasing the second path gain and it is more sensitive to second path delay than second path gain variations. 


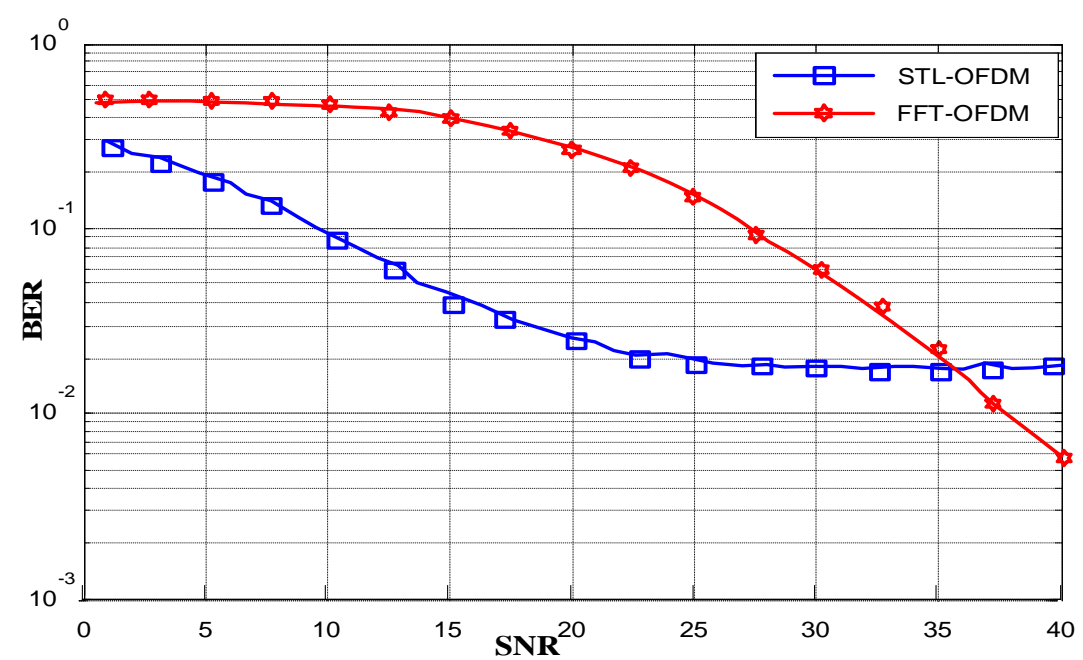

Fig. 12: The BER performance of SLT-OFDM in SFC at second Path Gain= $-11 \mathrm{~dB}$.

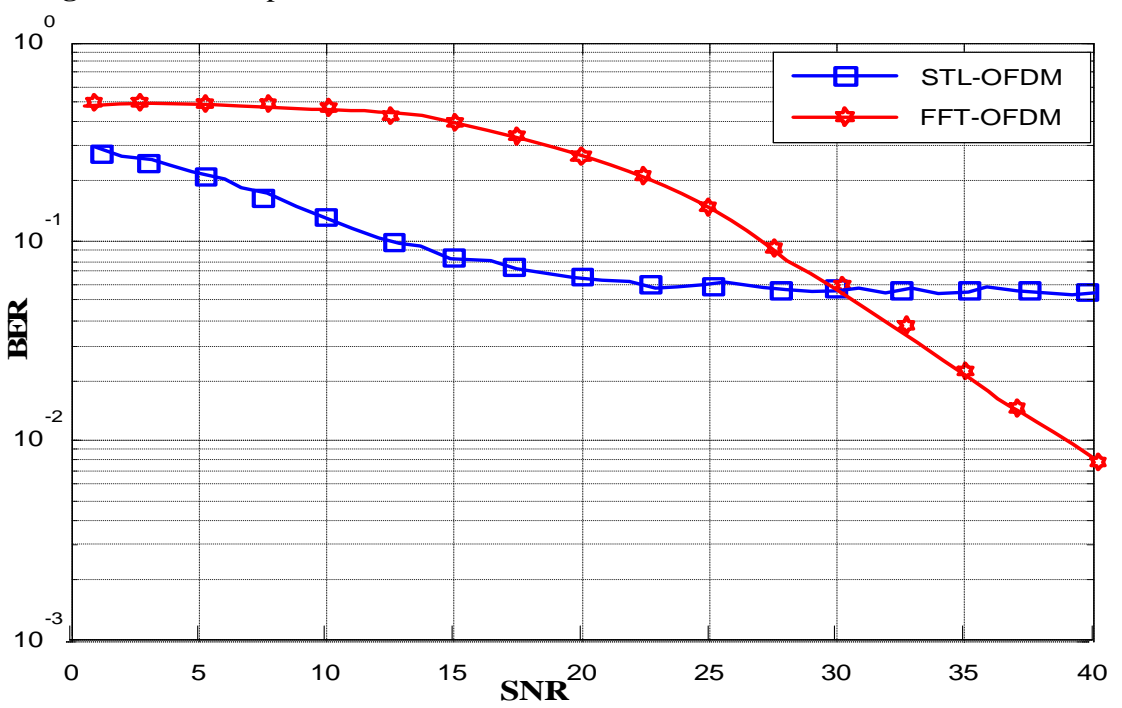

Fig. 13: The BER performance of SLT-OFDM in SFC at second Path Gain= $-5 \mathrm{~dB}$.

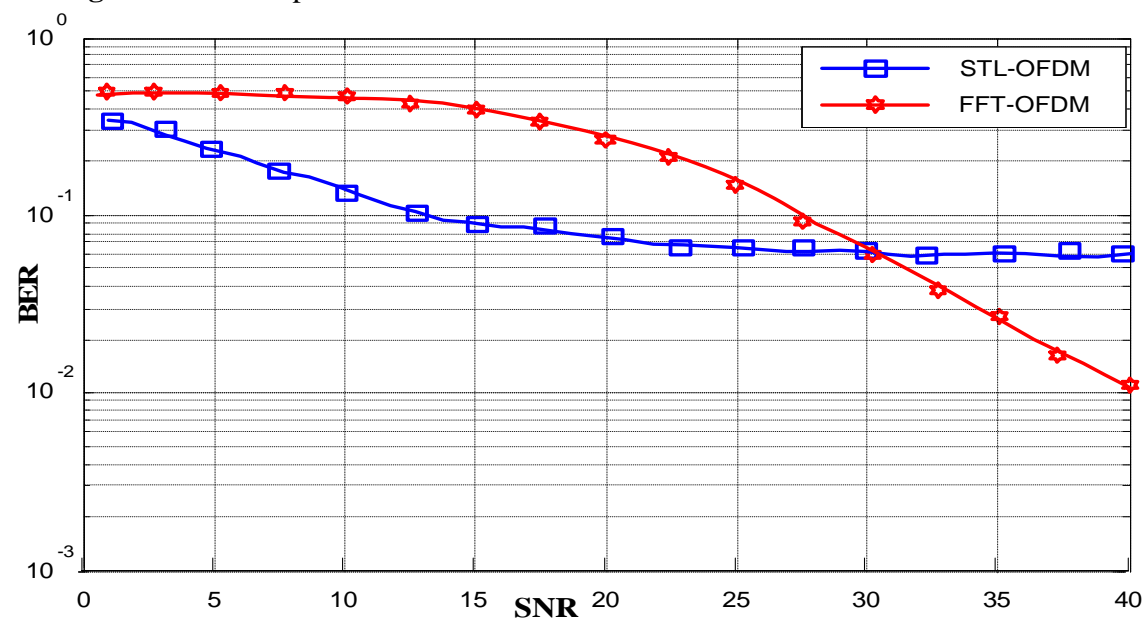

Fig. 14: The BER performance of SLT-OFDM in SFC at second Path Gain= $-1 \mathrm{~dB}$.

\section{Conclusions}

In this paper, the SLT-OFDM structure was proposed simulate and tested. Simulation results proved that; the proposed design achieves much lower bit error rates and better performance than FFT-OFDM assuming reasonable choice of the bases function and method of computations. Proposed SLT-OFDM gives in AWGN channel $\mathrm{BER}=10-4$ when $\mathrm{SNR}=17 \mathrm{~dB}$ while FFT based OFDM gives the same BER when $\mathrm{SNR}=33 \mathrm{~dB}$. In FFC and selective fading channels SLT based OFDM also has performance better than FFT-OFDM system. Proposed SLT-OFDM systems is robust for multi-path channels 
and does not require cyclically prefixed guard interval, which means that it obtains higher spectral efficiency than conventional OFDM and it can be used at high transmission rates. From obtained results it can be concluded, that SNR can be successfully increased using proposed SLT designed method. Therefore this structure can be considered as an alternative to the conventional OFDM.

\section{References}

[1] N. Al-Dhahir and J.M. Cioffi, "Optimum finite-length equalization for multicarrier transceivers", IEEE Trans. Communications, 44 (1996), pp. 56-64.

[2] S. Weinstein and P. Ebert, "Data Transmission by Frequency Division Multiplexing using the Discrete Fourier Transform”, IEEE Trans. Commun. Tech, COM-19 (1971), pp. 628-634.

[3] Nghi H. Tran, Ha H. Nguyen, and Tho Le-Ngoc, "BitInterleaved Coded OFDM with Signal Space Diversity: Subcarrier Grouping and Rotation Matrix Design“, IEEE Transactions on Signal Processing, 55 (2007), pp. 1137-1149.

[4] Won Gi Jeon, Kyung Hi Chang and Yong Soo Cho, "An Equalization Technique for Orthogonal Frequency-Division Multiplexing Systems in Time-Variant Multipath Channels", IEEE Transactions on Communications, 47 (1999), pp. 27-32.

[5] I. Koffman and V. Roman, "Broadband wireless access solutions based on OFDM access in IEEE 802.16”, IEEE Commun. Mag., 40 (2002), pp. 96-103.

[6] I. Lee, J.S. Chow, J.M. Cioffi, "Performance evaluation of a fast computation algorithm for the DMT in high-speed subscriber loop", IEEE J. Select. Areas Commun., 13 (2007), pp. 1564-1570.

[7] R. V. Nee and R. Prasad, "OFDM for Wireless Multimedia Communications", London, U. K.: Artech House, 2000.

[8] R. Prasad, "OFDM for Wireless Communications Systems", Artech House Publishers, 2004.

[9] L. J. Cimini Jr., "Analysis and simulation of a digital mobile channel using orthogonal frequency division multiplexing”, IEEE Trans. Commun., COM-33 (1985), pp. 665-675.

[10] SeungWon Kang and KyungHi Chang, "A Novel Channel Estimation Scheme for OFDM/OQAM-IOTA System", ETRI Journal, 29 (2007), pp. 430-436.

[11] IEEE Std.,"IEEE Proposal for 802.16.3, RM Wavelet Based (WOFDM) PHY Proposal for 802.16.3", Rainmaker Technologies, Inc., 2001.

[12] A.R. Lindsey, "Wavelet packet modulation for orthogonally multiplexed communication", IEEE Trans. Signal Process, 45 (1997), pp. 1336-1337.

[13] S. Mallat, "A Wavelet Tour of Signal Processing", second ed., Academic Press, New York, 1999.

[14] H.L. Resnikoff, J. Raymond, O. Wells, "Wavelet Analysis, The Scalable Structure of Information", Springer, New York, 1998.

[15] X. Zhang, P. Xu, G. Zhang, G. Bi, "Study on complex wavelet packet based OFDM modulation (CWP-OFDM)", ACTA Electron, Sinica, 30 (2002), pp. 476-479.

[16] H. Zhang, D. Yuan, M. Jiang, D. Wu, "Research of DFTOFDM and DWT-OFDM on different transmission scenarios", IEEE ICITA’2004, Harbin, China, 2004.

[17] H. Zhang, Dongfeng Yuan, Matthias Pätzold, "Novel study on PAPRs reduction in wavelet-based multicarrier modulation systems", Elsevier Inc, Digital Signal Processing,
17 (2007), pp. 272-279.

[18] Abbas Hasan Kattoush, Waleed. A. Mahmoud, S. Nihad, "The Performance of Multiwavelets Based OFDM System Under Different Channel Conditions", Elsevier Inc., DSP, doi:10.1016/j.dsp.2009.06.022.

[19] I. W. Selesnick, "The slantlet transform”, IEEE Trans. Signal Processing, 47 (1999), pp. 1304-1313.

[20] Edward R. Dougherty, Jaakko T. Astola, Karen O. Egiazarian "The fast parametric slantlet transform with applications" Proceedings of SPIE", 5298 (2004), pp. 1-12.

[21] G. Panda, P. K. Dash, A. K. Pradhan, and S. K. Meher "Data Compression of Power Quality Events Using the Slantlet Transform", IEEE Transactions on Power Delivery, 17 (2002), pp. 662-667.

[22] B. Alpert, G. Beylkin, R. Coifman, and V. Rokhlin, "Wavelet-like bases for the fast solution of second-kind integral equations”, SIAM J. Sci. Comput., 14 (1993), pp. 159184.

[23] I. Daubechies, "Ten Lectures on Wavelets", Philadelphia, PA: SIAM, 1992. 